Projets

de paysage

\section{Projets de paysage}

Revue scientifique sur la conception et l'aménagement de l'espace

$22 \mid 2020$

Forêt et paysage

\title{
La forêt et son imaginaire social : quels enjeux pour l'avenir?
}

The Forest and its Social Image: Future Challenges

\section{Yves Luginbühl}

\section{OpenEdition}

\section{Journals}

Édition électronique

URL : http://journals.openedition.org/paysage/7822

DOI : $10.4000 /$ paysage.7822

ISSN : 1969-6124

\section{Éditeur :}

École nationale supérieure du paysage de Versailles-Marseille, Institut national des sciences appliquées Centre Val de Loire - École de la nature et du paysage, École nationale supérieure d'architecture et de paysage de Bordeaux, École nationale supérieure d'architecture et de paysage de Lille, Agrocampus Angers

\section{Référence électronique}

Yves Luginbühl, «La forêt et son imaginaire social : quels enjeux pour l'avenir ? », Projets de paysage [En ligne], 22 | 2020, mis en ligne le 21 juillet 2020, consulté le 25 juillet 2020. URL : http:// journals.openedition.org/paysage/7822 ; DOI : https://doi.org/10.4000/paysage.7822 


\title{
La forêt et son imaginaire social : quels enjeux pour l'avenir?
}

\author{
The Forest and its Social Image: Future Challenges
}

Yves Luginbühl

1 Dans l'opinion publique la plus large, le terme de forêt renvoie à une étendue boisée plus ou moins vaste faite d'essences variées où les citadins vont se promener le dimanche en famille ou bien où l'on va chercher des champignons. D'une certaine manière, cette vision de la forêt correspond à peu près à la définition qu'en donne le Trésor de la langue française : "Vaste étendue de terrain couverte d'arbres; ensemble des arbres qui couvrent cette étendue.» Mais cette définition est large, peu précise et exclut la diversité immense des forêts qui couvrent une grande partie des continents : la forêt des pays scandinaves n'a rien à voir avec la forêt d'Amazonie ou celle de la péninsule ibérique, forêt souvent faite de chênes verts ou de chênes-lièges, dénommée dehesa Rien à voir non plus avec les forêts des Landes presque exclusivement composées de pins maritimes, ou avec celle de Fontainebleau, la première à être protégée en 1853 par un arrêté préfectoral à la suite des pressions du groupe des peintres de Barbizon. Et bien évidemment, de nombreux autres exemples existent sur la planète, comme les forêts de bambous chinoises ou de La Martinique, ou celles de palmiers à huile d'Indonésie, ou encore les « forêts » des oasis, faites de palmiers dattiers.

2 La forêt a toujours inspiré l'imaginaire des relations de l'homme à la nature. Selon les sociétés, la forêt occupe une place plus ou moins importante dans les représentations sociales des paysages selon que celui-ci est considéré comme bucolique, pittoresque, sublime ou romantique. Il s'agit de développer en premier lieu le sens que la forêt a occupé dans l'histoire des rapports sociaux à la nature dans plusieurs pays européens, comme par exemple la France et l'Allemagne qui entretiennent des relations très différentes à leur espace forestier.

3 L'imaginaire commun évoque souvent la forêt comme étant la principale et primitive occupation du sol dans l'histoire ; en particulier, le bocage, paysage fondé sur les arbres et les buissons regroupés en haies, serait un vestige d'une ancienne forêt. Or, il n'en est rien, du moins pour la France, sans doute davantage pour l'Allemagne, car le bocage est 
une construction issue de diverses règles d'occupation du sol et de décisions politiques. La forêt du célèbre personnage de bande dessinée Astérix ne s'est pas transformée en bocage par l'opération du Saint-Esprit, mais a dû être modifiée par arasement des boisements, puis édification de talus et semis ou plantations d'arbres sous forme de haies. Mais l'idée est restée solidement ancrée dans l'imaginaire populaire; il en est sans doute autrement en Allemagne où la forêt a alimenté de nombreux mythes qui se retrouvent dans la peinture romantique des paysages et dans la musique comme celle de Richard Wagner.

4 Après avoir retracé les principaux caractères de cette histoire symbolique de la forêt dans plusieurs pays européens, il s'agira d'approfondir les relations entre paysage, forêt et économie en prenant pour critère principal le bien-être ou le mal-être ressentis par les habitants proches des espaces forestiers. Ce critère du bien-être ou du mal-être a été utilisé dans la science économique pour évaluer les biens non marchands comme la biodiversité ou les services écosystémiques. Mais jusqu'à présent, le paysage n'a pas fait l'objet d'une analyse approfondie d'évaluation socioéconomique, sauf dans la vallée de la Loire, où un collectif de chercheurs et de paysagistes a pu évaluer le bien-être ou le mal-être ressentis par les habitants en réalisant des enquêtes par entretiens semidirectifs effectuées avec la Mission Val de Loire, organisme chargé de la gestion du site de la vallée de la Loire inscrit sur la liste du patrimoine mondial de l'Unesco.

Dans cette recherche, la forêt est cependant peu présente, elle apparaît par bribes et c'est pourquoi cet article se propose d'aller plus loin dans l'évaluation du bien-être ou $\mathrm{du}$ mal-être ressentis par les individus dans les espaces forestiers ou dans leur environnement proche. Car, ce qui apparaît souvent est le point de vue des habitants sur les arbres, que ces derniers soient regroupés en haies, bosquets ou boisements. Apparemment, les points de vue sont assez contradictoires, certains individus considérant que les boisements occultent la vue sur les paysages, d'autres au contraire estimant que la forêt constitue un critère de bien-être. C'est sur cette analyse que l'article se propose de revenir. Car il apparait que les individus ont une conscience claire et pertinente de ce que représente la biodiversité ou le changement climatique et du rôle que jouent la forêt et les arbres dans ces grands enjeux planétaires.

L'exemple historique des peintres de l'école de Barbizon (xıx siècle) est intéressant car il renvoie à la symbolique de la forêt, à son esthétique et aux sentiments qu'elle suggère auprès de ceux qui fréquentent la forêt de Fontainebleau. Cette conception ouvre sur la question vaste et fondamentale de l'imaginaire social de la forêt. Il s'agit d'un sujet immense, qui sera abordé dans la seconde partie de cette contribution après avoir retracé la situation autant économique, sociale et écologique de la forêt sur la planète, avec les enjeux qu'elle soulève et qui seront traités dans la troisième partie. Bien évidemment, entre ces trois sujets, il existe des interactions puissantes, car entre la question de l'aménagement des forêts et celle de son esthétique ou de sa symbolique, il existe de nombreux liens, comme il en existe également entre la symbolique et les enjeux économiques de la filière forestière.

7 Cette analyse doit permettre de considérer la forêt et les paysages auxquels elle contribue dans un contexte économique, en prenant le parti d'estimer le paysage comme un produit de l'économie; c'est à cette condition peu présente dans les recherches sur les paysages que l'on peut en effet évaluer le bien-être ou le mal-être que ceux-ci procurent à la société. Ces réflexions s'inscrivent dans la problématique plus générale de la politique actuelle qui renvoie à la place du citoyen dans la décision 
publique, telle que le "Grand Débat", organisé par le gouvernement français, l'a conçue à la suite de la crise démocratique et sociétale des gilets jaunes. Sans entrer dans le détail de cette crise, c'est bien dans ce contexte politique et économique que cette contribution placera ses conclusions.

\section{La forêt dans le monde : situation et évolution}

8 La première question concerne tout d'abord l'histoire des forêts européennes telles qu'elles apparaissent dès le Néolithique: leur fonction de refuge pour les tribus errantes à la recherche de gibier, de plantes comestibles et de bois pour se chauffer ou construire des huttes. La plupart du temps, ces populations aménageaient des clairières où elles construisaient des remparts de protection en pieux de bois prélevés sur la forêt alentour. Mais il faut se garder d'assimiler tout l'espace de vie de ces peuplades à la forêt; il existait également des steppes faites d'herbages naturels qui étaient utilisés pour nourrir le bétail. En Beauce, par exemple, il reste une croyance tenace consistant à penser que la Beauce était couverte de forêts, alors que cette région française n'était qu'un espace de steppe où les Romains installèrent des villas pour exploiter le sol, en utilisant les populations gauloises installées dans des villages proches, les vici. Les populations autochtones étaient pratiquement des esclaves travaillant dans les vastes domaines romains pour conduire les troupeaux et contribuer aux semailles et aux moissons.

9 Ailleurs, la forêt règne le plus souvent sur l'espace européen. Forêt largement sollicitée pour son bois, elle va connaître une diminution de ses superficies pendant plusieurs siècles, sauf à l'époque mérovingienne où les invasions barbares ont favorisé le développement de la forêt. Ce n'est que plus tard, au Moyen Âge, que la forêt s'est trouvée fortement sollicitée pour la construction, le chauffage, l'outillage, les forges, et la fabrication de véhicules.

On sait en particulier que la paysannerie a fortement contribué à la disparition de grands espaces forestiers en prélevant le bois pour se chauffer ou pour construire ses habitations, notamment. Parfois, la forêt était un espace collectif, c'est-à-dire des communaux, comme on en trouve encore dans la plus grande partie des pays européens. Mais les conflits entre les seigneurs ou les lords en Angleterre et les paysans à propos des terres communes ont entraîné des luttes souvent violentes, en particulier dans ce pays où les lords souhaitaient s'approprier ces commons pour en faire des terres d'élevage et édifier des enclosures entourant les champs (Hoskins, 1955); tout ceci au détriment de la forêt, qui, en Angleterre, a été rapidement mise à mal et s'est réduite à quelques domaines de l'aristocratie pour la chasse et les plaisirs des lords.

11 Toute la période du Moyen Âge au XviII ${ }^{\mathrm{e}}$ siècle a été une époque défavorable à la forêt, lourdement sollicitée par les prélèvements de la paysannerie, des seigneurs ou du clergé, comme par exemple en Bourgogne par l'ordre des Clunisiens qui firent construire le clos Vougeot avec de grandes poutres de chêne pour les logements des converts, ou pour construire les pressoirs avec du bois de chêne ${ }^{1}$. C'est au XVII siècle que les pouvoirs royaux ont commencé à prendre conscience de la pénurie de bois pour construire les navires commerciaux et militaires. Colbert créa les premières forêts royales, comme celle de Fontainebleau et celle de Tronçais grâce aux premières lois forestières de 1666. En même temps, Colbert souhaitait s'attaquer aux communaux pour les privatiser, mais la crainte des jacqueries l'a fait reculer. Ce n'est qu'à la 
Révolution française que fut votée une loi lors de la Convention, en juin 1791, pour supprimer les dits communaux; les résistances des paysans furent nombreuses et plusieurs régions, comme la Bourgogne, ont conservé les communaux avec la même superficie qu'à l'époque antérieure à la Révolution. En Italie, le projet de Cristoforo Sabbadino, ingénieur au service du doge de Venise au XVII e siècle, permit d'étendre la forêt sur les Beni inculti, terres des Dolomites plus ou moins en friche et collectives afin de produire les pieux sur lesquels reposent les nouveaux palais de la Sérénissime et le bois nécessaire à la construction des navires commerciaux et des galères militaires (Concina, 1992, p. 130-135).

12 C'est cependant à partir de la Révolution que la forêt a gagné du terrain en même temps que le bocage ${ }^{2}$. L'extension forestière est notamment due à l'action du ministre de l'Agriculture, de l'Intérieur et des Arts, Nicolas Louis François de Neufchâteau ${ }^{3}$, qui engagea un vaste programme de plantation d'arbres intitulé "Culture des arbres", consistant à planter dans tous les cantons de France des milliers d'arbres; des affiches étaient placardées dans toutes les préfectures du pays; le citoyen planteur était récompensé par une médaille d'or à son nom ou une stèle posée sur le lieu des plantations. En réalité, ce programme n'eut qu'un effet mineur et ce n'est que plus tard, en 1830, que, dans les Vosges, un programme de reconstitution des forêts fut engagé avec une prime au propriétaire de terrain qui assura la plantation des arbres; par ailleurs, le code forestier de 1827 permit également « une nouvelle réglementation de l'usage des forêts, en particulier concernant le ramassage du bois, les coupes et surtout le pâturage désormais mis en défens (interdit), le droit de marronnage, et les droits de chasse, de pêche et de cueillette ${ }^{4}$ ».

Cependant, malgré ces actions en faveur de la reconstitution des forêts, le développement industriel poursuivit les prélèvements de bois, pour les étais des mines de charbon en particulier, puis pour les traverses de chemin de fer; toute l'Europe fut concernée, surtout l'Angleterre dont la forêt était totalement exsangue. Mais le pays bénéficiait des enclosures où de nombreux chênes furent plantés, fournissant le bois nécessaire à la construction des navires, ce qui permit au Royaume-Uni de vaincre Napoléon en opposant un blocus maritime à la France. D'une manière générale, les surfaces forestières européennes ont régressé jusque vers la seconde moitié du XIX siècle, car c'est lors de cette période que les premières lois en faveur de la forêt furent votées dans de nombreux pays, comme la France ou l'Espagne. Ces lois provoquèrent des tensions entre les deux groupes d'ingénieurs forestiers, en France, les uns souhaitant un développement forestier rapide et sur les communaux, les autres défendant les droits des communautés paysannes sur les communaux. Le conflit entre le premier groupe et la paysannerie fut violent, notamment dans le Vercors où les ingénieurs forestiers firent appel à l'armée pour déloger les paysans occupant les terres communes : le conflit se solda par plusieurs dizaines de morts chez les paysans. Mais le conflit le plus connu est la fameuse affaire de la «Guerre des Demoiselles ${ }^{5}$ » qui se déroula dans les Pyrénées, en Ariège entre 1829 et 1832, et se prolongea jusqu'en 1872. Réaction sociale au code forestier de 1827, c'est le mouvement de contestation le plus connu parmi ceux qui se développent dans les Pyrénées au XIX siècle (Métailié, 2006). Il doit son nom au fait que les paysans révoltés s'habillaient avec des longues blouses blanches ou des peaux de mouton, des foulards ou des perruques, le visage noirci ou caché, pour attaquer - essentiellement la nuit - les grands propriétaires, les gardes 
forestiers et les gendarmes, les maitres de forges et les charbonniers, ce qui les faisait ressembler à des femmes.

En dépit de ces conflits entre les ingénieurs forestiers, représentant l'État, et les paysans, les lois forestières de la fin du XIX $\mathrm{X}^{\mathrm{e}}$ siècle ont peu à peu contribué à l'extension des forêts, mais ce fut également l'exode rural qui entraîna l'abandon de nombreuses terres par les agriculteurs, laissant ainsi la friche puis la forêt s'installer dans la plupart des zones de montagne. Puis le Fonds forestier national (FFN) fut créé en 1946 pour soutenir la filière bois et pour encourager le reboisement ${ }^{6}$; il a permis de mobiliser 32 milliards de Francs (6,3 milliards d'euros) pour aider les propriétaires à reboiser ; il fut supprimé en 2000, remplacé par une contribution volontaire qui alimente France Bois Forêt, interprofession regroupant les divers métiers de la filière bois-forêt.

Toujours est-il que la forêt française et, d'une manière générale, les forêts européennes ont connu une forte extension au $\mathrm{xx}^{\mathrm{e}}$ siècle. Mais la filière est peu organisée à l'échelle internationale, le commerce des bois est peu régulé, laissant de fortes importations de bois exotiques se faire au détriment des forêts tropicales, comme celles d'Afrique ou d'Amérique du Sud, en particulier celle d'Amazonie, dont les derniers événements sont d'une extrême gravité avec les incendies provoqués par les grands propriétaires brésiliens.

Ces derniers sont désireux d'étendre les cultures de soja transgénique ou de canne à sucre ou encore l'élevage, soutenus par le président du pays, Jair Bolsonaro, allié du président américain, tous les deux climatosceptiques. Si les forêts des pays dits développés, surtout en Europe, sont à peu près bien gérées, malgré l'extension des résineux ou d'essences inflammables comme l'eucalyptus au Portugal ou les pins dans les pays méditerranéens, il n'en est pas de même dans les pays tropicaux où les prélèvements de bois exotiques mettent à mal des forêts " primaires » comme la forêt atlantique du Brésil, celles du Congo, du Cameroun, etc. Il s'agit de pertes graves de surfaces forestières qui se font au détriment de la fixation du carbone et accélèrent le réchauffement climatique.

C'est l'arbre résineux qui attira les critiques les plus acerbes contre les reboisements de l'après-guerre, notamment dans le Limousin, les Ardennes et en Bretagne, à cause de l'acidification du sol et des sous-bois stériles, et surtout les formes de ces boisements dits en «timbres-poste», en raison des parcelles exiguës que les petits propriétaires plantaient avec l'aide de subventions du FFN. La France est dans une situation singulière, dans la mesure où il existe un nombre élevé de tout petits propriétaires forestiers dont les descendants ne savent même plus qu'ils ont hérité d'un petit boisement, ne connaissent pas sa localisation ni ses limites, ce qui pose un énorme problème à la gestion des forêts ; c'est pourquoi les Centres régionaux de la propriété forestière (CRPF) furent créés afin de regrouper les propriétaires de boisements et les aider à gérer leurs biens. La situation était d'autant plus difficile que dans certaines régions, comme le Massif central, les biens forestiers étaient des indivis ou des sectionnaux, dont la gestion était quasi impossible, notamment sur les cônes volcaniques où les parcelles forestières avaient moins de 1 mètre de largeur au sommet et plusieurs mètres à la base. C'est d'ailleurs pourquoi des regroupements fonciers ont été engagés pour résoudre ces problèmes de gestion. En tout cas, toutes ces questions à propos de l'évolution des forêts soulèvent celles de la symbolique et de l'imaginaire social qui lui ont été associés. 


\section{L'imaginaire social de la forêt}

18 La forêt est souvent ressentie comme un univers sombre, abritant des animaux sauvages, comme le loup du petit Chaperon rouge ou la célèbre bête du Gévaudan, terrible loup qui dévora tant d'imprudents s'aventurant dans la forêt. Donc, un lieu qui fait peur, redouté. Pendant longtemps, la forêt était également le refuge des bandits qui attaquaient les convois ou les diligences, comme on peut le voir dans de nombreux films de cape et d'épée. Les massifs forestiers ont aussi abrité les maquisards pendant la Seconde Guerre mondiale, notamment dans le Vercors ou sur le plateau des Glières, lieux de terribles combats entre la Résistance et les troupes allemandes du Reich nazi. Ce fut le cas également en Italie, dans le Piémont, où les résistants italiens luttèrent âprement contre l'armée de Mussolini au prix de nombreux morts ou de prisonniers. En France, la "Guerre des Demoiselles", conflit entre les populations locales et les ingénieurs forestiers est sans doute l'événement le plus connu des oppositions sociales aux reboisements.

19 Très tôt dans l'histoire, la forêt a suscité un imaginaire abondant précisément en raison de sa forme paysagère, faite du rassemblement d'arbres plus ou moins ouvert à la progression du marcheur, avec ou sans fourrés, souvent sombre, vallonné ou non, extrêmement varié en tout cas. Cet espace prisé des premiers peuples de l'époque néolithique était non seulement un refuge, mais également un réservoir à gibiers, à bois et à quelques plantes comestibles comme les champignons. Il était investi de nombreuses croyances, parfois lieu de regroupement de créatures imaginaires comme les elfes ; à l'époque gauloise, la forêt était aussi le lieu des rassemblements des druides qui se consacraient à des rites religieux avec le gui sacré recueilli de préférence sur les branches d'un chêne. Certains lieux de la forêt étaient sacralisés, comme les sources ou les temples, édifices nommés fanum ou nemeton, selon les modes de construction.

20 À toutes les époques et dans toutes les cultures, la forêt a entretenu des légendes; même dans les civilisations éloignées de la France, les arbres de la forêt pouvaient posséder des pouvoirs extraordinaires, parfois les femmes y venaient en recherchant un arbre qui pouvait, selon les croyances, leur assurer la fécondité. Et en absence de forêt, comme en Afghanistan, pays aride et de hautes montagnes où l'arbre est rare, les camionneurs peignent sur les flancs de leur véhicule des paysages verdoyants avec des lacs bleus où nagent des cygnes et dans le fond du tableau de vertes forêts. De même, au Népal, les forêts qui entourent les premiers versants de l'Himalaya ${ }^{7}$ ont des vertus que connaissent les peuples installés sur les pentes impressionnantes des hautes montagnes : vertus médicinales en particulier. Dans ce pays, les autorités ont décidé de protéger les forêts qui appartiennent à la famille royale, aux dépens des populations montagnardes. Or, ces ethnies avaient un fort besoin de bois, pour se chauffer ou construire leurs maisons ; leur réaction fut localement innovante, car les populations de ces villages ont inventé leur propre bocage en plantant des frênes le long des talus des terrasses cultivées (Smadja, 2003).

21 Malgré la rareté des forêts dans le Royaume-Uni, les arbres ont suscité la production de textes poétiques, en particulier à propos des chênes des enclosures qui ont servi à bâtir les navires de la flotte anglaise ; ainsi le poète Pope vante-t-il la beauté des chênes des enclosures; la Windsor Forest de Pope constitue ainsi un emblème incarnant la souveraineté politique et culturelle britannique dont le symbole est le chêne, espèce préférée des artisans au xvIII siècle $^{8}$. Assurant le fondement matériel du commerce 
anglais et de sa suprématie maritime, le chêne est l'essence constitutive des navires suggérant ces phrases au poète britannique :

"While by our Oaks the precious loads are born,

And realms commanded which those Trees adorn. »

Cependant, c'est dans la comparaison entre la mythologie de la forêt allemande et celle des autres pays que l'on évalue au mieux les différences de l'imaginaire social forestier en Europe. Contrairement à l'imaginaire forestier des pays comme la France ou les pays méditerranéens, où la forêt est plutôt marquée par la peur ancestrale d'y rencontrer des animaux sauvages et bien que la situation ait fortement changé avec la fréquentation dominicale des forêts périphériques aux grandes villes par les familles, en Allemagne, la forêt revêt presque toujours un caractère positif. Les mythes de la forêt allemande sont nombreux, à commencer par Siegfried ${ }^{9}$ qui combat le dragon (Fáfnir dans la mythologie nordique) et le tue, puis il se baigne dans son sang pour être invulnérable ; mais il ne s'est pas rendu compte qu'une feuille de tilleul est tombée sur son dos, entre les omoplates, faiblesse qui conduit à anéantir le pouvoir fourni par le sang du dragon. Lorsque Siegfried combat son ennemi Gupormr ${ }^{10}$, il est prêt à le vaincre, mais un traitre a averti son ennemi de la fragilité du héros allemand qui tombe, mort, à cause d'un coup d'épée sur la trace de la feuille de tilleul. Et c'est bien la forêt allemande qui est le théâtre de ce drame.

C'est également dans la peinture allemande que se révèle cette mythologie singulière : les peintres allemands de la période romantique aiment à peindre le clair-obscur, c'està-dire la Dunkelheit, terme qui traduit une profonde affection pour les rayons du soleil tombant en oblique sur la forêt et tout particulièrement sur les grottes où se réfugient les ermites. Il existe de nombreuses toiles de peintres allemands qui représentent ce clair-obscur. 
Figure 1. Johann Heinrich Füssli, Siegfried terrassant le dragon, 1790

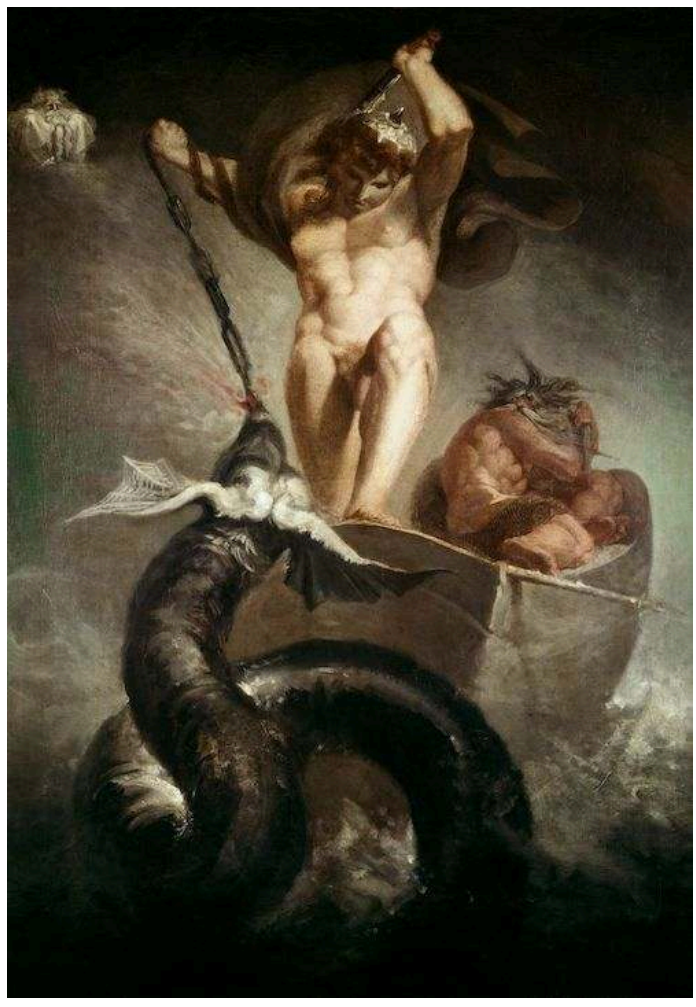

$50 \times 71 \mathrm{~cm}$, huile sur toile.

Source : domaine public 
Figure 2. Carl Blechen, Paysage rocheux avec moine, 1825

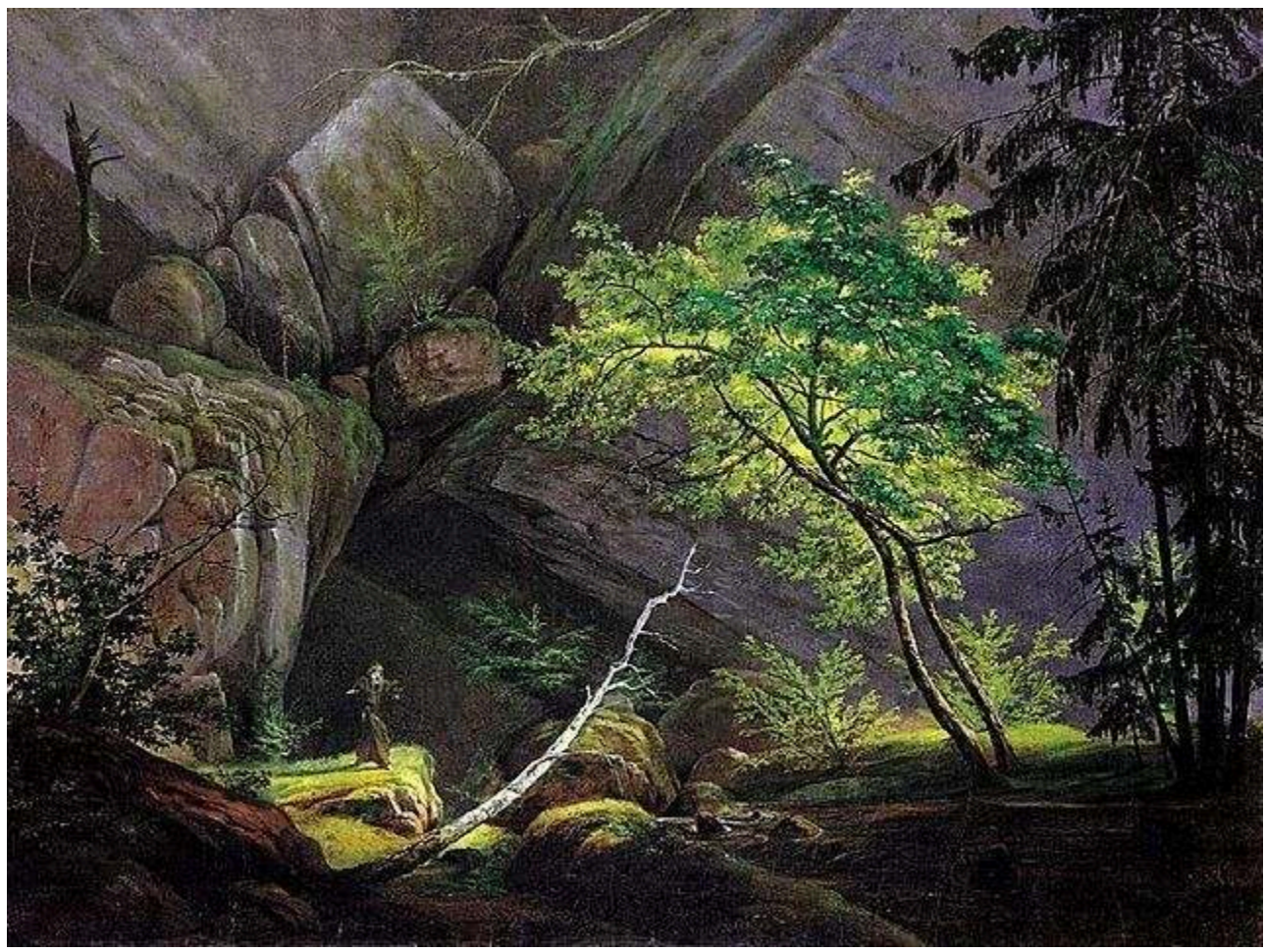

Huile sur toile, $70 \mathrm{~cm}$ x $94 \mathrm{~cm}$, conservée à la Galerie nationale de Berlin.

Source : domaine public.

24 Adolescent, j'allais souvent en Allemagne, en bordure de la Forêt-Noire ; avec mes amis allemands, nous allions presque tous les soirs, à la tombée de la nuit, nous promener dans la forêt, et revenions au clair de lune; cette anecdote confirme l'affection de la société allemande pour cette atmosphère sombre de la forêt; par ailleurs, ces amis m'emmenaient souvent nous baigner dans les étangs du bord du Rhin, la nuit, où tous les Allemands, en famille, se baignaient nus. Le naturisme est très pratiqué dans ce pays, en particulier la nuit comme le confirme le film du réalisateur Luchino Visconti Les Damnés, avec Helmut Berger, où les soldats SA se baignent dans un lac dans une atmosphère de clair-obscur, au clair de lune, avant de se faire assassiner par les SS lors de la nuit des Longs Couteaux.

25 Toujours est-il que la différence avec la conception française ou méditerranéenne de la forêt par rapport à celle de l'Allemagne est fortement marquée notamment par la présence dans la première de la recherche de la lumière. C'est en tout cas ce que l'on peut constater chez les peintres impressionnistes valorisant la lumière, même dans leurs représentations de la forêt, avec le chêne de Fontainebleau de Claude Monet ou les pins sur la montagne Sainte-Victoire de Paul Cézanne. 
Figure 3. Paul Cézanne, Montagne Sainte-Victoire, 1887

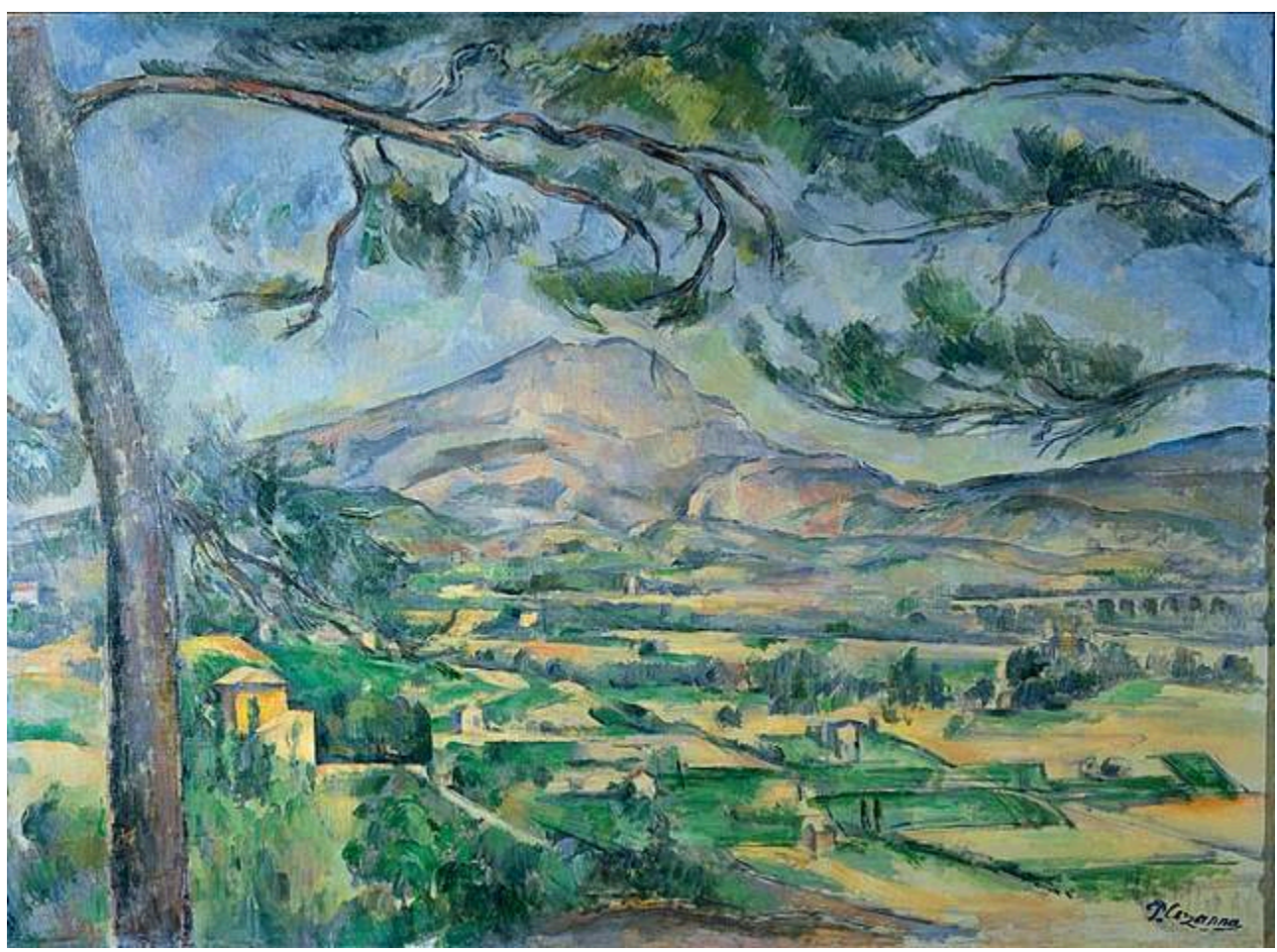

Conservé au Courtauld Institute of Art, Londres.

Source : domaine public.

Ailleurs, comme en Espagne, la forêt est valorisée par de nombreux usages. C'est le cas de la dehesa, forêt claire de chênes verts ou chênes-lièges taillés régulièrement pour aérer les boisements et produire du charbon de bois. Ce sont ces superbes forêts que l'on trouve en Andalousie ou en Estrémadure ou encore dans les sierras proches de Madrid. Elles portent le mythe d'un système écologique complet, c'est-à-dire agrosylvopastoral, permettant de produire des céréales sous couvert forestier, du bois et d'élever des animaux, en particulier les porcs de race ibérique bien connus pour fournir le célèbre jamon de pata negra, sans doute l'un des meilleurs du monde. Ces porcs se nourrissent des glands des chênes à l'époque de la glandée, puis de racines ou d'herbes diverses. Leur graisse est composée d'un acide gras saturé comme celui de l'huile d'olive, ne produisant pas de cholestérol. Le cycle cultural de ces dehesas alterne ainsi production céréalière, fourragère, animale et forestière, certains écologues les considèrent comme un modèle agro-écologique accompli de biodiversité, ce qui reste pourtant à démontrer. 
Figures 4. Forêt claire en Andalousie, une dehesa

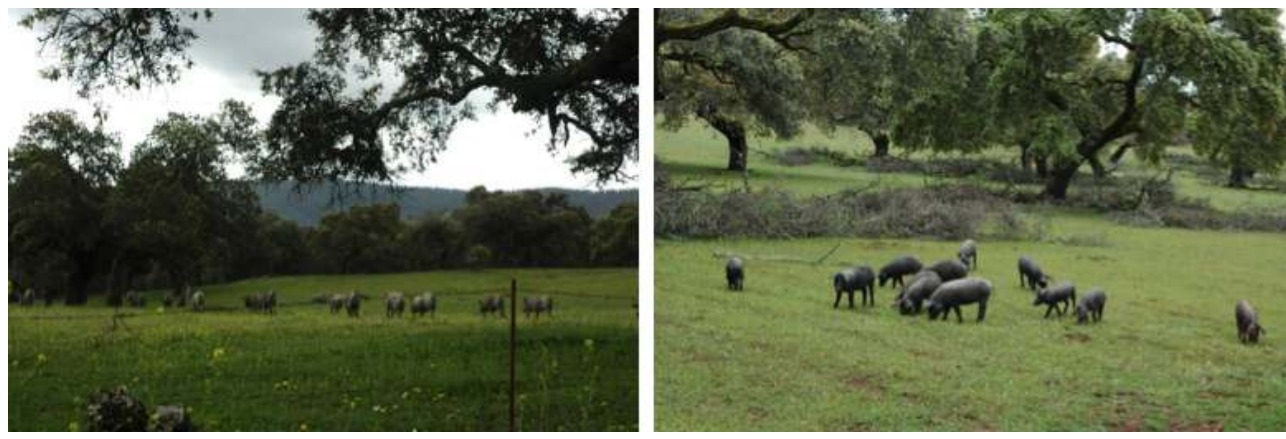

Cette forêt est faite de chênes verts et de chênes-lièges, avec les porcs de race ibérique dits de pata negra.

Source : clichés de l'auteur.

Il serait possible de multiplier les exemples de ces visions quasi mythiques des forêts ; je ne peux m'empêcher de citer la forêt d'Amazonie, évoquée précédemment pour les récents événements d'incendies extrêmement graves, mais qui contient aussi un imaginaire social puissant : forêt peuplée d'ethnies qui ont fait leur renommée grâce au grand ouvrage de Claude Lévy-Strauss, Tristes Tropiques (1955), où il analyse les pratiques et les croyances des Amérindiens de cette forêt ; superbe étude qui montre encore davantage le pouvoir imaginaire de la forêt chez un peuple apparemment fruste mais pourtant combien riche de ses usages culturels de la nature.

Ces questions renvoient à la valeur esthétique de la forêt : les modèles esthétiques du paysage sont surtout liés au paysage rural, beaucoup moins à celui de la forêt; si le paysage rural est souvent considéré comme pastoral ou bucolique et encore pittoresque, les paysages de la montagne et des littoraux sont davantage sublimes ou parfois pittoresques également. La forêt n'entre pas toujours dans ces modèles, mais les peintres de Barbizon ont estimé que la forêt de Fontainebleau était pittoresque, c'est-àdire propre à être peinte. Sans doute pourrait-on envisager les grandes forêts tropicales comme sublimes, car sujettes à susciter la peur et la capacité de leurs visiteurs à la surmonter.

\section{Les enjeux du développement forestier dans l'avenir et la question du bien-être}

L'intérêt pour la forêt oscille donc entre la peur du sauvage, la fascination pour ses mythes et l'idéologie dont elle peut être porteuse. Maintenant, il s'agit de savoir comment ces forêts, d'une extrême diversité d'un bout à l'autre de la planète, peuvent trouver un développement propice à la biodiversité et à la qualité de vie des peuples qui y vivent, en vivent ou qui en sont proches. Rien ne dit pour le moment, qu'en raison des risques du réchauffement climatique et de l'érosion de la biodiversité, ces forêts trouvent une voie idéale pour de tels objectifs, d'autant plus que, comme mentionné plus haut, le marché international du bois est fortement aléatoire, sans véritable régulation. Si, globalement, les forêts européennes s'accroissent, comme la forêt française en raison de l'abandon de terres par l'agriculture, il n'en est pas de même des forêts tropicales qui voient leurs superficies régresser régulièrement, car les bois exotiques sont fortement exploités et exportés vers les pays riches. Et par ailleurs, l'on 
sait que ces forêts tropicales sont souvent détruites par leur mise en culture. Les enjeux sont donc considérables car les forêts fixent le $\mathrm{CO}_{2}$ et émettent de l'oxygène, contribuant ainsi à la lutte contre le réchauffement climatique.

Jusqu'à présent, l'économie ne s'est pas toujours beaucoup intéressée à la forêt, sauf pour évaluer leur valeur écologique. Cependant, l'économie forestière représente un secteur important des activités économiques à l'échelle planétaire, car elle représente un marché mondial d'échanges et de transactions, sans véritable régulation. De nombreux pays africains ou d'Amérique du sud ou d'Asie exportent des bois exotiques à forte valeur, mettant à mal le patrimoine forestier des pays concernés et dégradent leur biodiversité. Or, l'évaluation socioéconomique des aménités, c'est-à-dire des biens non marchands, n'a pas toujours eu les résultats que l'on pouvait escompter. En particulier, les analyses contingentes ou évaluations des coûts monétaires parviennent à des montants qui n'expriment pas vraiment la valeur d'un bien non marchand comme le paysage ou le patrimoine. Les économistes hétérodoxes ont d'ailleurs critiqué ces méthodes (y compris celles des coûts de déplacement) et ont cherché à introduire une dimension quantitative dans ces évaluations. Un progrès assez considérable a été fait avec le rapport Stiglitz, du nom de l'économiste américain prix Nobel d'économie qui a remis au président de la République, Nicolas Sarkozy, en 2008, un document réalisé avec Amartya Sen et Jean-Paul Fitoussi sur les performances économiques de la France (Stiglitz, Sen et Fitoussi, 2008). Dans ce rapport, les auteurs insistent sur la nécessité d'introduire des critères qualitatifs et en particulier celui du bien-être objectif et subjectif :

《Recommandation $\mathrm{n}^{\circ} 10$ : Les mesures du bien-être, tant objectif que subjectif, fournissent des informations essentielles sur la qualité de la vie. Les instituts de statistiques devraient intégrer à leurs enquêtes des questions visant à connaître l'évaluation que chacun fait de sa vie, de ses expériences et priorités. » (Ibid, p. 16.)

31 C'est à partir de ces avancées qu'une équipe de chercheurs et de paysagistes s'est penchée sur l'évaluation socioéconomique du bien-être et du mal-être procurés par le paysage. Jusqu'alors, ce type d'analyse tentait plutôt d'évaluer une aménité, comme le paysage en utilisant des critères divers tels que le bien-être. Le postulat de départ de cette recherche réalisée sur le Val de Loire avec la Mission Val de Loire, organisme chargé de la gestion du site inscrit sur la liste du patrimoine mondial de l'Unesco est le suivant: le paysage est un produit de l'économie et il s'agit d'évaluer le bien-être ou le mal-être procurés par le paysage qui évolue au gré des transformations de l'économie (Mission Val de Loire, 2015). L'équipe de recherche a réalisé environ 85 entretiens semidirectifs auprès de deux échantillons de population de deux secteurs du Val de Loire, l'un proche de Châteauneuf-sur-Loire et l'autre près de Trélazé, ces deux parties de la vallée présentant des caractères paysagers et économiques relativement différents. Les questions portaient surtout sur les lieux qui, pour les personnes interrogées, procuraient $\mathrm{du}$ bien-être ou à l'inverse du mal-être; le résultat a été au-delà des attentes de l'équipe de recherche, car les habitants ont parfaitement intégré la question du bien-être ou du mal-être. Mais ce qui intéresse cet article, c'est bien évidemment la question de la forêt. Or, celle-ci est assez peu présente sur ces deux secteurs, mais suffisamment pour fournir des résultats peu communs. Non seulement il a été possible d'établir des cartes permettant de localiser les lieux de bien-être ou de mal-être, mais en outre, les personnes interrogées ont pu identifier 52 critères de bien-être ou de malêtre, se référant à des domaines de l'économie comme l'emploi, l'artisanat ou l'industrie, les infrastructures, l'éducation, la santé, le logement, la construction, le 
patrimoine, etc. ; et parmi ces domaines, celui de l'environnement avec la perception de la biodiversité et des boisements. Dans ces boisements présents sur le Val de Loire, la forêt de Sologne marque la limite du secteur est de la recherche; cette forêt n'est pas forcément un lieu de bien-être pour les habitants qui regrettent les clôtures à l'intérieur de la forêt, en raison de grandes propriétés souvent réservées à la chasse. Mais par ailleurs, ils reconnaissent que la forêt de Sologne offre un cadre agréable à proximité de Sully-sur-Loire :

« Ah oui, moi j'aime tout ce qui est campagne, bois, forêt : aller dans le bois derrière pour entendre les oiseaux, voir les écureuils, ça fait du bien... » (un habitant de Sully-sur-Loire).

« "... sur Orléans, c'est une forêt, au niveau de la Loire, moi, je pense que les boucles de la Loire, c'est un paysage, les perspectives que l'on peut avoir sur la Loire, c'est magnifique » (un habitant de Saint-Benoit-sur-Loire).

Ce n'est pas toujours une forêt telle que l'on peut l'imaginer, épaisse, sombre, mais ici, c'est parfois un boisement de peupliers qui trouble les résidents. En effet, sur les bords de la Loire, de nombreux peupliers ont poussé, même sur les îles, masquant les vues sur la Loire. C'est ce qui s'est passé près de Châteauneuf-sur-Loire, là où, d'habitude, les habitants venaient sur la rive contempler le fleuve et en particulier assister le 14 juillet au feu d'artifice de la fête nationale ; avec leur croissance, les peupliers interdisent de bien voir ce feu d'artifice et les habitants le regrettent; ils ont protesté auprès de la mairie qui, finalement, a fait abattre les peupliers, permettant ainsi la vision de la manifestation festive depuis les bords de Loire. Ailleurs, un ancien pâturage a été recolonisé par la forêt, en bord de la Loire, et les habitants considèrent cette forêt humide comme un lieu de mal-être alors qu'auparavant, ils pouvaient voir les moutons paître tranquillement dans les près.

L'approche par le bien-être ou le mal-être est donc intéressante parce qu'elle permet de localiser les paysages forestiers où les habitants se sentent bien ou mal. Elle permet également d'identifier des critères de bien-être ou de mal-être par rapport à divers secteurs de la vie économique. Dans le contexte du réchauffement climatique, elle apporte de nouveaux enseignements, en particulier sur les risques d'incendies de forêts. Ainsi, dans la vallée de la Loire, Météo France a réalisé une étude qui permet d'évaluer ces risques à l'horizon 2060.

Figure 5. Cartes des risques d'incendies de forêts, en 2008 et projection en 2060
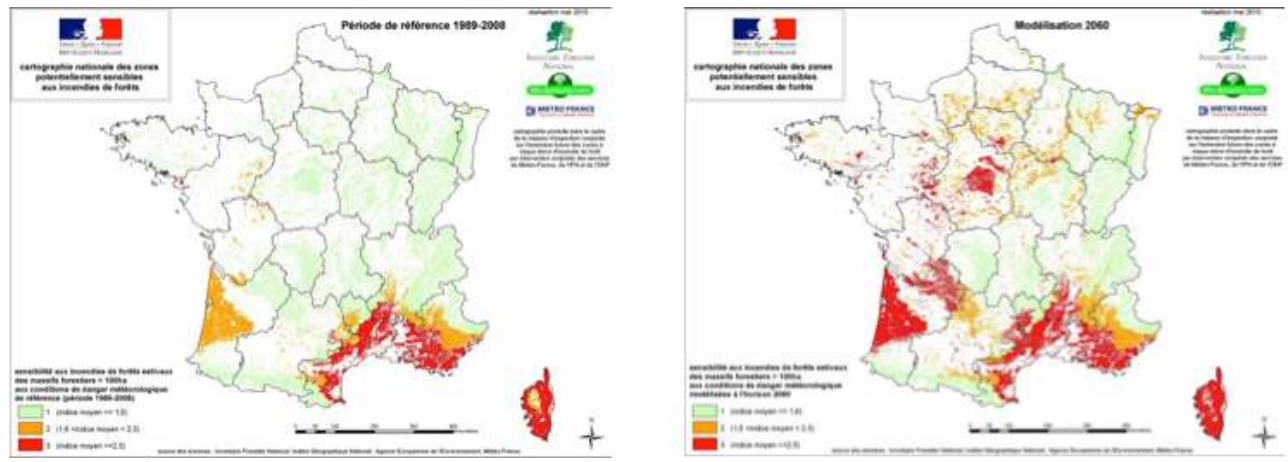

Sources : Daniel Vendramini de Météo-France et Emmanuelle Crépeau du PNR Anjou-Touraine. 
Ces risques d'incendies sont également liés à la migration des espèces forestières, telle que le révèlent les mêmes auteurs pour la France : ils ont évalué les diminutions et les régressions de ces espèces sur un délai de 120 ans.

Figure 6. Cartes des groupements forestiers français

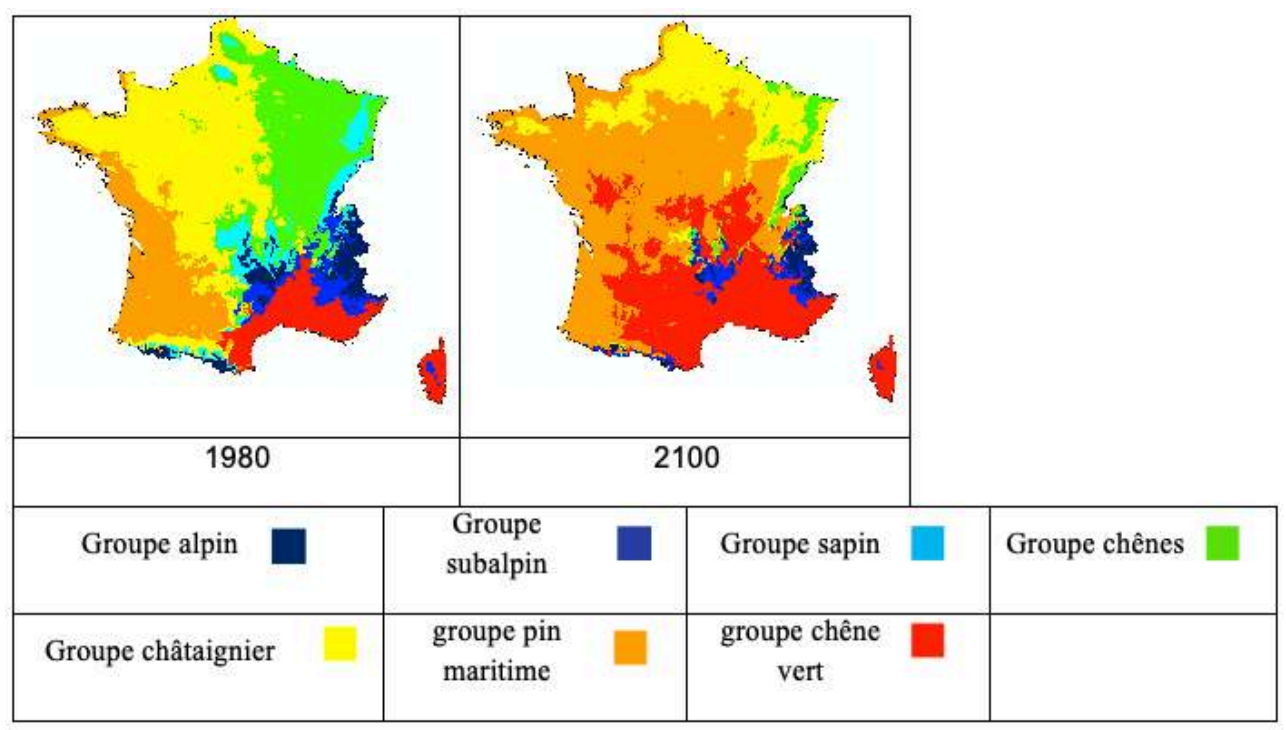

Sources : Daniel Vendramini de Météo-France et Emmanuelle Crépeau du PNR Anjou-Touraine.

Mais il faut être vigilant car les migrations d'espèces végétales peuvent être dues à l'intervention humaine. Par exemple, le chêne vert dont les écologues prédisent la remontée vers le Nord, a été implanté au XIX siècle dans les îles d'Aran, proches de l'Écosse ; c'est le duc d'Hamilton, qui accueillit Napoléon III pendant son exil, qui planta dans son parc quelques exemplaires de cette espèce. Ce n'est d'ailleurs pas le seul exemple de l'action humaine dans les migrations d'espèces forestières. Cependant, les espèces animales migrent également: en particulier les oiseaux qui nichent dans la forêt ; dans un petit bois de Bretagne est arrivé un jour un couple de huppes fasciées (Huppupa Epops) qui est resté environ un mois dans la forêt où il avait établi son nid. On pouvait les entendre chanter de ce chant tout à fait caractéristique et on les voyait picorer les vers de terre dans l'herbe. Habituellement, cette espèce était cantonnée au sud de la Loire, mais le réchauffement climatique lui a permis de migrer vers le Nord. 
Figure 7. Huppe fasciée

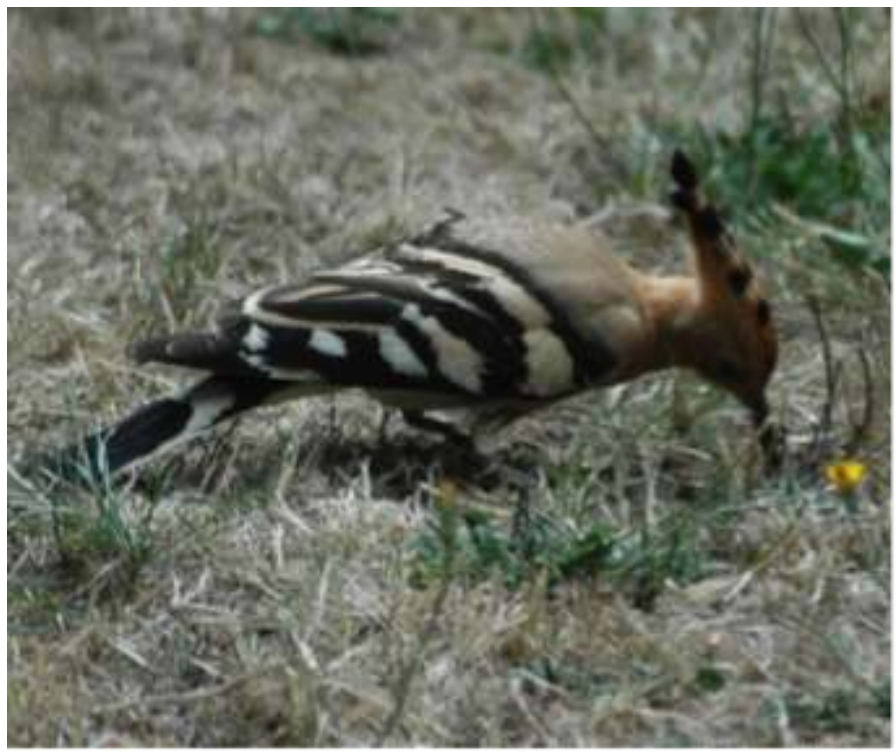

Elle s'est installée dans un jardin, nichant dans le bois limitrophe, sur la commune de Névez, Finistère. Source : cliché de l'auteur.

Bien des espèces végétales sont concernées par le réchauffement climatique, à commencer par les espèces invasives (Rémy et Beck, 2008, p. 193-209). Dans la vallée de la Loire, les peupliers sont de plus en plus souvent remplacés par les érables négondos, considérés par les spécialistes comme espèces envahissantes. Ces arbres posent des problèmes car les castors ne s'y attaquent plus alors qu'ils font leurs terriers avec le bois des peupliers. Ainsi, les érables négondos prolifèrent et vont à l'encontre de la biodiversité dont l'érosion constitue un enjeu planétaire. D'ailleurs, le réchauffement climatique et l'érosion de la biodiversité vont de pair, notamment à propos des forêts du monde entier.

L'approche socioéconomique par le bien-être ou le mal-être permet d'identifier des critères se référant à presque tous les secteurs de l'économie, que ce soit l'emploi, la santé, l'éducation, les infrastructures, l'urbanisme, etc. Avec ces critères, il est possible d'élaborer des indicateurs sociaux de bien-être ou de mal-être, pour tout paysage et, dans ce cas précis, pour les paysages forestiers.

\section{Conclusion}

Le paysage forestier est tout d'abord d'une extrême diversité. Il est impossible de comparer la taïga sibérienne à la dehesa espagnole ou aux forêts tropicales d'Afrique ou d'Amérique du Sud. Cependant, toutes les forêts rendent un service indispensable à la planète en fixant le carbone et en émettant de l'oxygène ; c'est pourquoi la régression des forêts à l'échelle planétaire est si préoccupante pour l'humanité. La diminution des superficies forestières est due tout d'abord aux incendies, comme en Amazonie ou en Indonésie, en Californie et même en Europe, mais cette diminution n'est pas partout la même. Dans certains pays, la forêt a vu sa surface augmenter, comme en France où elle occupe désormais plus du quart du territoire national en raison de l'abandon des terres par l'agriculture ; c'est aussi le cas dans plusieurs pays d'Europe. Ce qui ne signifie pas qu'il ne faut pas être vigilant, bien évidemment. En France, par exemple, les incendies 
de forêt ont régressé, mais il suffit de peu pour qu'ils se déclenchent à nouveau. La subsistance des forêts est primordiale pour lutter contre les effets du réchauffement climatique ou l'érosion de la biodiversité.

Cela dit, cette importance des superficies forestières n'est pas étrangère à l'imaginaire social que les forêts ont suscité. Comment analyser ces imaginaires sociaux? C'est en recueillant les représentations sociales des paysages forestiers auprès de leurs usagers que l'on peut analyser les manières dont ces paysages sont perçus. Les premières enquêtes sur la forêt ont eu lieu dans les années 1970 en France lorsque les grandes villes comme Paris et sa banlieue se sont étendues au détriment des terres agricoles et des forêts et que les habitants de ces métropoles commençaient à fréquenter les forêts pour leurs loisirs (Ballion, 1975). Les forêts représentaient alors l'un des seuls lieux que les populations urbaines pouvaient fréquenter les jours de congés hebdomadaires, ce qui a fortement contribué à forger une image positive de ce paysage dans les représentations sociales. Il en est de même pour d'autres pays comme l'Espagne où, aux environs des grandes villes comme Madrid ou Séville, les habitants allaient et vont encore se promener ou se délasser dans les forêts proches. Une enquête réalisée dans les bois de pins près de Séville auprès des Domingueros ${ }^{11}$, terme consacré aux promeneurs du dimanche, révéla que c'était le seul lieu où ils pouvaient s'adonner au pique-nique, faire des barbecues pour le déjeuner en famille et jouer avec leurs enfants au football, parfois en groupes relativement importants réunissant plusieurs familles. En fait, cette fréquentation des forêts périurbaines compensait un manque cruel de parcs de loisirs ou l'impossibilité de fréquenter la campagne alors tenue par une agriculture latifondiaire avec d'immenses parcelles et souvent closes, surtout dans les sierras andalouses couvertes de dehesas. Celles-ci étaient systématiquement entourées de clôtures de barbelés pour maintenir le bétail dans les propriétés.

40 La forêt a donc peu à peu changé de statut social, passant du lieu du risque, du danger, des animaux sauvages à un lieu de fréquentation hebdomadaire pour les loisirs, agréable et procurant du bien-être. Et c'est grâce à cette démarche que l'on peut développer une évaluation socioéconomique du paysage forestier en se fondant sur le postulat que le paysage est un produit de l'économie et que les activités économiques transforment les paysages et qu'en retour, les évolutions de ceux-ci modifient les représentations que s'en font les acteurs sociaux. Certes, cette démarche est nouvelle parmi les méthodes d'évaluation des aménités, comme le paysage, mais elle a donné des résultats lors de la recherche effectuée par la Mission Val de Loire et une équipe de chercheurs et de paysagistes déjà mentionnée. Les 52 critères de bien-être et de malêtre se réfèrent à de nombreux secteurs de l'économie. C'est à partir de ces critères que l'on peut élaborer des indicateurs de bien-être ou de mal-être permettant d'infléchir les politiques économiques.

41 Cela dit, il est alors nécessaire de mettre en relation cette approche avec les politiques forestières. Comme indiqué précédemment, le marché du bois n'est pas régulé à l'échelle mondiale; conduire une politique forestière exige donc une attention particulière vis-à-vis des acteurs concernés ; par exemple, une politique forestière peut consister à utiliser les produits des coupes non nobles pour alimenter des chaudières à bois afin de contribuer à la transition énergétique; c'est notamment ce qu'a entrepris la ville de Dijon avec deux centrales thermiques à déchets de bois pour chauffer plusieurs quartiers et produire de l'électricité. Mais la récupération des déchets de bois n'est pas toujours aisée : ainsi, dans le Morvan, les agriculteurs qui taillent les haies 
entourant leurs parcelles d'élevage de charolais rechignent à stocker les résidus de la taille parce que cette opération leur prend du temps et de l'énergie. C'est donc un produit perdu pour la transition énergétique. Pourtant, ce type d'opération fonctionne bien ailleurs, comme en Normandie où un éleveur utilise les déchets de la taille des haies pour alimenter une chaudière qui chauffe sa maison et ses bâtiments d'élevage ${ }^{12}$. La transition énergétique demande donc que les politiques forestières soient articulées avec d'autres politiques, par exemple celle du logement, soit pour le chauffage, soit même pour la construction; si les maisons en bois se développent de plus en plus en Europe, elles sont loin d'atteindre le niveau des États-Unis où la très grande majorité des maisons individuelles est construite en bois. Au Chili également, dans les campagnes, de nombreuses maisons sont en bois, parfois sur pilotis. Toutes les politiques d'aménagement du territoire peuvent être articulées avec les politiques forestières, mais cette relation n'est que très peu utilisée ; c'est donc dans ce sens que les recherches doivent se diriger, tout en accentuant les analyses des représentations sociales des paysages forestiers, de manière à nourrir la connaissance des imaginaires sociaux de la forêt.

\section{BIBLIOGRAPHIE}

Ballion, R. « La fréquentation des forêts ", Revue forestière française, t. XXVII, janvier 1975. Brun, A. et Chaize, M.-C. « Le paysage forestier, analyse des préférences du public », Doc INRA, n 13,1976 .

Concina, E., « La Renaissance : Venise, le territoire, le paysage », dans Luginbühl, Y. (dir.), Paysage méditerranéen. Catalogue de l'exposition sur le paysage méditerranéen, Milan, Electa, 1992, p. 130-135.

Corvol, A. et al. (textes choisis et présentés), Regards sur la forêt française, Paris, L’Harmattan, Paris, 2014.

Corvol, A. (textes réunis et présentés), Tempêtes sur la forêt française, $\mathrm{XVI}^{e}-\mathrm{XX} \mathrm{X}^{e}$ siècle, Paris, L'Harmattan, 2005.

Ferry, L., Le Nouvel Ordre écologique. L'arbre, l'animal et l'homme, Paris, Le Livre de poche, 2002, $221 \mathrm{p}$.

Fourneau, F., Roux, B. et Vacher, J., Supervivencia de la Sierra Norte de Sevilla, Evolución de los paisajes y ordenación del territorio en Andalucia Occidental, Madrid, Publications de la Casa de Velazquez, 1986, 376 pages.

Hoskins, W.G., The making of the English landscape, Londres, Hodder and Stoughton, 1955.

Kalaora, B., « Un loisir urbain, la forêt de Fontainebleau », Études rurales, no 83, juilletseptembre 1981.

Lévy-Strauss, C., Tristes Tropiques, Paris, Plon, coll. « Terre Humaine », 1955, 490 p. 
Luginbühl, Y., Fourneau, F. et Roux, B., Évolution des paysages, transformations socio-économiques et aménagement du territoire en Andalousie occidentale, Madrid, Publications de la Casa de Velázquez, 1991

Métailié, J.-P., « La dégradation des montagnes au XIX siècle dans les Pyrénées ", dans Beck, C., Luginbühl, Y. et Muxart, T., Temps et Espaces des crises de l'environnement, Versailles, Quæ, coll. «Indisciplines », 2006.

Mission Val de Loire, « Les dynamiques des paysages du Val-de-Loire », rapport, 2015, UMR Citères (université de Tours), UMR Ladyss, CNRS, Paris1, 7, 8 et 10, Cemotev (université de SaintQuentin-en-Yvelines), Passeurs Paysagistes, financé par le FEDER et la Région Centre Val de Loire, 174 pages.

Rémy, E. et Beck, C., «Allochtone, autochtone, invasive : catégorisations animales et perception d'autrui », Politix, vol. 21 nº 82, 2008, p. 193-209.

Smadja, J., Histoire et Devenir des paysages en Himalaya. Représentations des milieux et gestion des ressources au Népal et au Ladakh, Paris, Éditions du CNRS, coll. « Espaces et Milieux », 2003, 646 p.

Stiglitz, J., Sen, A. et Fitoussi, J.-P., « Rapport de la Commission sur la mesure des performances économiques et du progrès social ", 2008, URL : https://www.vie-publique.fr/sites/default/files/ rapport/pdf/094000427.pdf.

\section{NOTES}

1. Au clos Vougeot, trois pressoirs existent encore, faits de lourdes poutres et de vis de chêne ; ils furent construits au XVIII ${ }^{\mathrm{e}}$ siècle, alors que la partie abritant les chaix et les habitats des ouvriers datent du XVI ${ }^{\mathrm{e}}$.

2. Grâce notamment à l'instauration de la propriété individuelle du sol.

3. Nicolas Louis François de Neufchâteau, dit plus brièvement François de Neufchâteau, fut incarcéré pendant la Terreur et échappa de justesse à la guillotine. Il tenta d'engager également des actions de remembrement.

4. Il s'agit surtout de la rupture de l'équilibre entre le système traditionnel agro-sylvo-pastoral pyrénéen et le système technico-économique de propriétaires s'appuyant sur le code forestier et désirant intensifier le charbonnage afin d'alimenter principalement l'industrie, c'est-à-dire les forges établies le long des cours d'eau descendant des Pyrénées.

5. Voir : https://fr.wikipedia.org/wiki/Guerre_des_Demoiselles\#mw-head

6. Créé par la loi du 30 septembre 1946 en application du rapport Leloup de mai 1945 (selon les vœux du Conseil national de la Résistance); un autre outil a été créé également : l'Inventaire forestier, créé le 24 septembre 1958. Cet organisme a fusionné avec l'IFN en 2012. L'inventaire est aujourd'hui réalisé par l'Institut national de l'information géographique et forestière.

7. C'est de ces forêts que vient la majorité des espèces de rhododendrons cultivées en Europe.

8. Ce n'est pas un hasard si, après d'autres essences, le chêne s'est imposé comme l'essence forestière dominante des enclosures $\mathrm{au} \mathrm{XVIII} \mathrm{e}^{\mathrm{e}}$ siècle. Auparavant la politique maritime britannique était aussi fondée sur l'exploitation radicale des forêts anglaises qui ont fortement diminué ; le système des enclosures constitue un moyen de renouveler le stock d'arbres destinés à la construction de navires.

9. Sigurd (Sigurðr en vieux norrois, Siegfried en allemand) est un héros légendaire de la mythologie nordique qui apparaît dans plusieurs poèmes héroïques de l'Edda poétique, compilés au XIII ${ }^{\mathrm{e}}$ siècle. La tétralogie de Richard Wagner, Der Ring des Nibelungen, qui s'inspire des 
traditions allemandes et scandinaves pour créer une œuvre originale, a largement contribué au regain d'intérêt de la légende de Siegfried dans le grand public.

10. Dans la mythologie nordique et non allemande, cette histoire de Sigurd est très complexe et mêle de nombreux personnages. La version allemande a été simplifiée.

11. Enquête réalisée par une équipe de recherche de la Casa de Velázquez de Séville lors d'un programme de recherche consacré à l'évolution des paysages d'Andalousie occidentale (Luginbühl et al., 1991).

12. Exploitation agricole de Normandie, près de Picauville.

\section{RÉSUMÉS}

La forêt a toujours nourri, de manière féconde, l'imaginaire des rapports de l'homme à la nature. Selon les sociétés, la forêt occupe une place plus ou moins importante dans les représentations sociales des paysages selon que ces derniers sont considérés comme bucolique, pittoresque, sublime ou romantique. L'article se propose de développer en premier lieu le sens que la forêt a occupé dans l'histoire des rapports sociaux à la nature dans plusieurs pays européens, comme par exemple la France et l'Allemagne qui entretiennent des relations très différentes à leur espace forestier. Après avoir retracé les principaux caractères de cette histoire symbolique de la forêt dans plusieurs pays européens, l'article se propose d'approfondir les relations entre paysage, forêt et économie en prenant pour critère principal le bien-être ou le mal-être ressentis par les habitants proches des espaces forestiers.

The forest has always been a fertile source of inspiration which has nourished the imagination of humankind and its relationship to nature. In different societies, depending on whether the forest is perceived as a bucolic, picturesque, sublime or romantic element, its role in the social representations of the landscape takes on more or less importance. The article seeks to focus on the significance of the forest in the history of social relationships to nature in several European countries, such as France and Germany for example, and which differ greatly from country to country. After describing the main features of this symbolic history of the forest in several European countries, the article examines more closely the relationships between the landscape, the forest, and the economy with as the main criterion the sense of well-being or unease experienced by people living close to forests.

\section{INDEX}

Mots-clés : paysage, forêt, bien-être, économie, participation citoyenne

Keywords : landscape, forest, well-being, economy, citizen participation

\section{AUTEUR}

\section{YVES LUGINBÜHL}

Yves Luginbühl est directeur de recherche émérite au CNRS, UMR Ladyss, CNRS, universités de Paris1, 7, 8 et 10. Ses domaines de recherche sont le paysage, l'environnement, l'aménagement du 
territoire, la biodiversité et les interactions entre les processus sociaux et les processus biophysiques.

yves.luginbuhl[at]univ-paris1[dot]fr 